\title{
Metformin in combination with rosiglitazone contribute to the increased serum adiponectin levels in people with type 2 diabetes mellitus
}

\author{
JIE-MING NIE ${ }^{1}$ and HAI-FENG LI ${ }^{2}$ \\ ${ }^{1}$ Department of General Medicine, The Third Affiliated Hospital of Guangzhou Medical University, Guangzhou, \\ Guangdong 510150; ${ }^{2}$ Department of Pharmaceutical Analysis, ALK-Abello A/S Guangzhou Office, \\ Guangzhou, Guangdong 510620, P.R. China
}

Received March 28, 2017; Accepted July 12, 2017

DOI: $10.3892 /$ etm.2017.4823

\begin{abstract}
To evaluate how metformin plus rosiglitazone affect serum adiponectin levels in people suffering from type 2 diabetes mellitus (T2DM), 240 patients having T2DM were selected in this cohort study. Included subjects were randomly and equally separated into three subsets: i) Group A (rosiglitazone group); ii) group B (metformin group); and iii) group $\mathrm{C}$ (rosiglitazone + metformin group). Furthermore, meta-analysis of previous studies was performed by searching the general search engines and bibliographic databases. Compared with before treatment, the serum amount of adiponectin grew considerably in the three groups after treatment, and the levels in the group $\mathrm{C}$ was much greater than those of groups $\mathrm{A}$ and $\mathrm{B}$ (all $\mathrm{P}<0.05$ ). Corresponding meta-analysis results suggested post-treatment serum adiponectin level to be greater than pretreatment level in $\mathrm{T} 2 \mathrm{DM}$ patients $(\mathrm{P}<0.001)$. Further subgroup analyses indicated that combination therapy of metformin and rosiglitazone may increase the amount of serum adiponectin in T2DM sufferers among the majority subgroups (all $\mathrm{P}<0.05)$. The combination of metformin and rosiglitazone treatment increased serum adiponectin levels, suggesting that metformin plus rosiglitazone therapy is a suitable choice to treat T2DM.
\end{abstract}

\section{Introduction}

Metformin is medically considered as the only biguanide which is used and recommended as oral anti-diabetic agent, which is crucial for decreasing the levels of plasma glucose (1).

Correspondence to: Dr Hai-Feng Li, Department of Pharmaceutical Analysis, ALK-Abello A/S Guangzhou Office, Room 903, B2 Building, Site 5 of 99, Jiang Nan Da Dao Road, Haizhu, Guangzhou, Guangdong 510620, P.R. China

E-mail: lcwayy@163.com

Key words: adiponectin, type 2 diabetes mellitus, metformin, rosiglitazone
As known, metformin has been found to exert an increasing effect on inhibiting hepatic gluconeogenesis, decreasing hyperinsulinemia, reducing protein synthesis, improving insulin sensitivity and enhancing glucose use in the muscle $(2,3)$. Metformin may therefore be applicable for a wide use for the treatment of metabolic syndrome, reducing the risk of cancer and delaying aging (4). In clinical practice, previous evidence has reported that metformin is widely accepted as an effective treatment for diabetes mellitus (DM), and notably to type $2 \mathrm{DM}$ (T2DM) by serving as the first-line therapy (5). Apart from metformin, rosiglitazone is well-known as another valuable anti-diabetic drug which is a member of the thiazolidinedione class of drugs (6). Studies have shown that rosiglitazone has the ability to attenuate inflammatory effects, elicit insulin sensitivity and lower the glucose by serving as the peroxisome proliferator-activated receptor- $\gamma$ (PPAR- $\gamma$ ) agonists $(7,8)$. Accordingly, rosiglitazone has an obvious effect on atherosclerosis, inflammation, endothelial dysfunction and T2DM (9).

Adiponectin may be capable of modulating the processes of lipid metabolism and regulating glucose (10). Abundant previous evidence has shown that the lack or dysfunction of adiponectin was proved to be intensely correlated with insulin resistance due to its characteristic as an insulin-sensitizing hormone $(11,12)$. It was well established that T2DM is not only a disease of glucose metabolism, but an inflammatory disease, inflammatory factors have been suggested to be critically implicated (13-15). The abnormal lipid metabolism in patients with diabetes can aggravate the inflammatory reaction. Importantly, adiponectin has been identified to be important for properties as anti-inflammation, insulin-sensitivity and anti-atherosclerosis $(16,17)$. Plasma adiponectin levels present a negative correlation with insulin sensitivity; therefore, it decreases when insulin resistance and diabetes develop $(18,19)$. In addition, adiponectin may result in the reduction of the content of intracellular cholesteryl ester in human macrophages (20). With respect to the above, an obvious increase in adiponectin may suggest the curative effect of metformin and glimepiride in treatment of T2DM.

Metformin has been found to stimulate the secretion of adiponectin and to upregulate the expression of adiponectin 
to some extent (21). Thus, it is shown that the metformin has a certain association with serum adiponectin levels in people suffering from T2DM (22). On the other hand, using the rosiglitazone to treat patients with T2DM may be able to stimulate the production of adiponectin in adipocytes, which means that there may be a certain association between the rosiglitazone and the serum adiponectin levels in patients with T2DM (23). Importantly, recent investigation has shown that therapy of patients with T2DM through metformin as well as rosiglitazone may upregulate serum adiponectin levels obviously by reducing the insulin resistance greatly, so the treatment may be valuable and important to the patients with T2DM (24). To this day, numerous studies have been published indicating that using metformin in combination with rosiglitazone may be a promising treatment regimen for patients with T2DM by affecting the serum adiponectin levels $(25,26)$ while other studies had illustrated contradictory results $(27,28)$. Therefore, we conducted the present investigation on the basis of a clinic-based cohort study and meta-analysis to determine the association between the combined treatment of metformin plus rosiglitazone and the serum adiponectin levels in people suffering from T2DM.

\section{Materials and methods}

Ethics statement. Our research process carried out with the presence of human participants conformed to the ethical requirements of the Research Committee of The Third Affiliated Hospital of Guangzhou Medical University (approval no. 2012003) and to the Declaration of Helsinki 1964 (https:// www.mendeley.com/research-papers/world-medical-asssociation-declaration-helsinki/) and subsequent amendments. Each participant signed a comprehensive consent form before the investigation.

Subjects. Altogether 240 patients suffering from T2DM were selected and admitted to this cohort study from January 2012 to July 2014. Diagnosis criteria of diabetes was predefined based on the criteria in 2007 by the American Diabetes Association (29) included subjects met the requirements set out below: Fasting plasma glucose $(\mathrm{FPG}) \geq 7.0 \mathrm{mmol} / \mathrm{l}$, or glycosylated haemoglobin A1c (HbA1c) $\geq 6.5 \%$, or oral glucose tolerance test (OGTT) measured $2 \mathrm{~h}$ blood glucose level $\geq 11.1 \mathrm{mmol} / 1$, or patients with typical high blood sugar or high-blood sugar crisis showed an obvious trend for an increase in random blood glucose level $\geq 11.1 \mathrm{mmol} / 1$. In addition, in patients without clear hyperglycemia, repeated detection of FBG, HbA1c and OGTT measured $2 \mathrm{~h}$ blood glucose were used. There were 164 males and 76 females, aged 32-76 years, with a mean age of $50.50 \pm 9.19$ years. The duration of disease was 0.4-23.0 months (mean value of $11.40 \pm 6.55$ years). Patients were enrolled based on the following criteria: i) All patients were free of chronic liver disease; ii) had no hyperthyroidism and other endocrine and metabolic diseases; iii) without bone joint diseases, or bone metastasis cancer affecting calcium and phosphorus metabolism; iv) without severe systemic disease; v) without ketoacidosis or a long time history of bed rest; vi) without hormone, vitamin $\mathrm{D}_{3}$ and calcium medication history; vii) without administration history of other agents irrelevant to anti-diabetic properties to exclude the influence to serum levels of adiponectin; and viii) selected female patients were post-menopausal women for 10 years. The included subjects were separated at random into three subsets ( 80 patients in each set) and managed by different treatment regimens. Data generators were used to populate tables with random test data from 1 to 240 , counts in multiples of three was subdivided into group A, and the remaining numbers in multiples of two was subdivided into group B, and the last remaining numbers were subdivided into group $\mathrm{C}$, who were treated with rosiglitazone, metformin and rosiglitazone + metformin, respectively.

Clinical data collection and related cytokine detection. Fasting elbow venous blood samples $(5 \mathrm{ml})$ were taken from the subjects in the morning, followed by centrifugation at $2,250 \mathrm{x}$ g for $10 \mathrm{~min}$, serum samples were isolated at $4^{\circ} \mathrm{C}$ and followed by plasma glucose and lipid analyses. Furthermore, samples were saved at $-20^{\circ} \mathrm{C}$ for the measurement of serum adiponectin. All indexes were measured at the beginning of treatment (pretreatment) and 24 weeks after treatment (posttreatment). Body mass index (BMI), waist circumference, waist/hip ratio, FPG, HbAlc, 2 h blood glucose, triglyceride, cholesterol, high-density lipoprotein (HDL), low-density lipoprotein (LDL), alanine aminotransferase, aspartate aminotransferase, serum total bilirubin and direct bilirubin of all the studied subjects were measured. The levels of adiponectin was examined enzyme-linked immunosorbent assay (ELISA) kit according to the manufacturer's instructions.

Treatment regimens. All the included patients received dietary intervention and exercise therapy, other drugs affecting plasma glucose of the patients were avoided during the experiment. Medication: i) Group A (rosiglitazone group), taking rosiglitazone (4 mg; GlaxoSmithKline, Middlesex, UK), once a day; ii) group B (metformin group), taking metformin ( $0.15 \mathrm{~g}$; Beijing Zhonghui Pharmaceutical Co., Ltd., Beijing, China) 3 times a day, the medicines were taken during or after meal to reduce gastrointestinal reactions; and iii) group $\mathrm{C}$ (rosiglitazone + metformin group), taking $4 \mathrm{mg}$ rosiglitazone once a day and $0.15 \mathrm{~g}$ metformin 2 times a day. Medicine in the three groups were all oral administered lasting for 24 weeks.

Data sources and retrieval strategies. Before March 2016, available literature, assessing the effect of metformin combined with rosiglitazone on serum amount of adiponectin in T2DM sufferers, were obtained via the application of computer datasets and search engines (Embase, Web of Science, PubMed and China National Knowledge Infrastructure). 'Metformin' or 'Dimethylguanylguanidine' or 'Glucophage' or 'Diabex' or 'Dimethylbiguanide' or 'Melbin' or 'Mellitin' or 'Hydrochloride' as well as 'rosiglitazone' or 'rosiglitazone maleate' or 'Avandia' or 'BRL 49653' or 'BRL49653' or 'BRL-49653' as well as 'Adiponectin' or 'adiponectin' or 'Adipocyte Complement-Related Protein 30-kDa' or 'Adipose Most Abundant Gene Transcript 1' or 'ACRP30 Protein' as well as 'Diabetes Mellitus' or 'diabetes' or 'diabetic mellitus' or 'mellitus' or 'type 2 diabetes mellitus' or 'type 1 diabetes mellitus' or 'type 1 diabetes' or 'type 2 diabetes' were utilized as the mesh items and key words. Only articles published in Chinese and English were eligible. 
Table I. Comparisons of basic information among groups.

\begin{tabular}{|c|c|c|c|c|c|c|}
\hline \multirow[b]{2}{*}{ Variables } & \multicolumn{2}{|c|}{ Group A } & \multicolumn{2}{|c|}{ Group B } & \multicolumn{2}{|c|}{ Group C } \\
\hline & Pretreatment & Post-treatment & Pretreatment & Post-treatment & Pretreatment & Post-treatment \\
\hline FPG (mmol/l) & $7.22 \pm 1.62$ & $6.64 \pm 1.40^{\mathrm{a}}$ & $7.29 \pm 1.74$ & $6.60 \pm 1.35^{\mathrm{a}}$ & $7.32 \pm 1.60$ & $6.24 \pm 1.32^{\mathrm{a}-\mathrm{c}}$ \\
\hline HbA1c $(\%)$ & $7.02 \pm 1.12$ & $6.54 \pm 0.94^{\mathrm{a}}$ & $7.09 \pm 1.03$ & $6.49 \pm 0.95^{\mathrm{a}}$ & $7.08 \pm 1.04$ & $6.16 \pm 0.89^{a-c}$ \\
\hline $2 \mathrm{~h}$ blood glucose $(\mathrm{mmol} / \mathrm{l})$ & $15.67 \pm 2.72$ & $13.57 \pm 2.61^{\mathrm{a}}$ & $15.20 \pm 2.43$ & $13.40 \pm 2.47^{\mathrm{a}}$ & $15.42 \pm 2.23$ & $11.28 \pm 2.34^{\mathrm{a}-\mathrm{c}}$ \\
\hline Triglyceride (mmol/l) & $5.66 \pm 1.78$ & $5.51 \pm 1.45^{\mathrm{a}}$ & $5.58 \pm 1.50$ & $5.43 \pm 1.43^{\mathrm{a}}$ & $5.62 \pm 1.61$ & $5.12 \pm 1.56^{\mathrm{a}-\mathrm{c}}$ \\
\hline Cholesterol (mmol/l) & $2.44 \pm 1.30$ & $2.21 \pm 0.90^{\mathrm{a}}$ & $2.36 \pm 1.26$ & $2.17 \pm 0.88^{\mathrm{a}}$ & $2.36 \pm 1.33$ & $2.01 \pm 0.85^{\mathrm{a}-\mathrm{c}}$ \\
\hline $\mathrm{HDL}(\mathrm{mmol} / \mathrm{l})$ & $1.34 \pm 0.35$ & $1.22 \pm 0.32^{\mathrm{a}}$ & $1.38 \pm 0.37$ & $1.24 \pm 0.30^{\mathrm{a}}$ & $1.35 \pm 0.33$ & $1.13 \pm 0.25^{\mathrm{a}-\mathrm{c}}$ \\
\hline $\mathrm{LDL}(\mathrm{mmol} / \mathrm{l})$ & $3.48 \pm 1.27$ & $3.23 \pm 1.05^{\mathrm{a}}$ & $3.46 \pm 1.25$ & $3.19 \pm 1.12^{\mathrm{a}}$ & $3.42 \pm 1.35$ & $3.11 \pm 1.00^{\mathrm{a}-\mathrm{c}}$ \\
\hline Alanine aminotransferase (mmol/l) & $46.33 \pm 20.54$ & $32.81 \pm 14.57^{\mathrm{a}}$ & $45.57 \pm 19.69$ & $29.79 \pm 13.77^{\mathrm{a}}$ & $43.68 \pm 21.50$ & $24.62 \pm 14.21^{\mathrm{a}-\mathrm{c}}$ \\
\hline Aspartate aminotransferase $(\mathrm{mmol} / \mathrm{l})$ & $32.32 \pm 15.62$ & $23.90 \pm 7.49^{\mathrm{a}}$ & $31.66 \pm 14.21$ & $24.04 \pm 7.45^{\mathrm{a}}$ & $31.22 \pm 15.06$ & $20.05 \pm 6.90^{\mathrm{a}-\mathrm{c}}$ \\
\hline Serum total bilirubin (mmol/l) & $28.68 \pm 13.60$ & $14.56 \pm 4.54^{\mathrm{a}}$ & $26.90 \pm 12.57$ & $13.61 \pm 4.24^{\mathrm{a}}$ & $27.47 \pm 14.14$ & $10.44 \pm 4.02^{\mathrm{a}-\mathrm{c}}$ \\
\hline Direct bilirubin (mmol/l) & $3.88 \pm 1.38$ & $2.78 \pm 1.27^{\mathrm{a}}$ & $3.75 \pm 1.30$ & $2.72 \pm 1.24^{\mathrm{a}}$ & $3.76 \pm 1.34$ & $2.51 \pm 1.15^{\mathrm{a}-\mathrm{c}}$ \\
\hline BMI $\left(\mathrm{kg} / \mathrm{m}^{2}\right)$ & $28.32 \pm 6.72$ & $28.11 \pm 6.90$ & $28.81 \pm 7.23$ & $28.26 \pm 6.83^{\mathrm{a}}$ & $28.76 \pm 6.80$ & $27.34 \pm 5.52^{\mathrm{a}-\mathrm{c}}$ \\
\hline Waist circumference $(\mathrm{cm})$ & $93.19 \pm 7.70$ & $91.44 \pm 14.57$ & $92.48 \pm 8.03$ & $90.42 \pm 7.56^{\mathrm{a}}$ & $93.13 \pm 7.90$ & $88.21 \pm 6.76^{\mathrm{a}-\mathrm{c}}$ \\
\hline Waist/hip ratio & $0.98 \pm 0.05$ & $0.95 \pm 0.06$ & $0.94 \pm 0.07$ & $0.91 \pm 0.06^{\mathrm{a}}$ & $0.96 \pm 0.06$ & $0.86 \pm 0.05^{\mathrm{a}-\mathrm{c}}$ \\
\hline Adiponectin (ng/ml) & $12.08 \pm 6.38$ & $21.98 \pm 6.56^{\mathrm{a}}$ & $12.83 \pm 4.55$ & $22.51 \pm 7.28^{\mathrm{a}}$ & $12.26 \pm 5.68$ & $26.26 \pm 7.52^{\mathrm{a}-\mathrm{c}}$ \\
\hline
\end{tabular}

${ }^{\mathrm{a} C}$ Compared to the values before treatment; ${ }^{\mathrm{b}}$ compared to the values in group A; ${ }^{\mathrm{c}}$ compared to the values in group B. HbA1c, glycosylated haemoglobin A1c; HDL, high-density lipoprotein; LDL, low-density lipoprotein; BMI, body mass index.

Inclusion criteria were: i) Studies had to be human-associated studies; ii) designed in a cohort type; iii) had to provide available and adequate data considering serum adiponectin level pre- and post-treatment of metformin and rosiglitazone; and iv) without overlapping data with other studies. And when two or more studies were conducted by the same authors, only the latest and complete study met our consideration standards.

Information extraction and quality estimation. Two researchers (J-M Nie and H-F Li) extracted information separately relied on a standard form, and arrived at a consensus, when there was disagreement, with the third investigator who was specializing in this field. A set of predefined criteria was used to evaluate enclosed research, namely, Critical Appraisal Skills Programmer (CASP) criteria (http://www.casp-uk. net/\#!casp-tools-checklists/c18f8).

Statistical analysis. Power analysis was carried out by the Power and Sample Size Calculation (PS) program, we computed the needed sample sizes for a 0.80 probability of determining influence at the predefined level (i.e., $\alpha=0.05$ ) for statistical power analysis. A sampling of 240 estimable people for each treatment group would yield nearly $80 \%$ power to determine effects at the predefined level (i.e., $\alpha=0.05$ ) in groups A-C. Regarding clinical experimental data analysis, statistical analyses were done using SPSS 19.0 statistical software (SPSS, Inc., Chicago, IL, USA). Continuous data are presented as mean \pm standard deviation, and t-test was used to confirm the results. For meta-analysis, summary standard mean differences (SMDs) with 95\% confidence interval (CI) were used for statistical comparison with the utilization of $\mathrm{Z}$ test, which was aggregated utilizing the STATA software, version 12.0 (StataCorp, College Station, TX, USA).

\section{Results and Discussion}

Benchmark traits. In group A, there were 56 males as well as 24 females, having a mean age of $52.21 \pm 12.62$ years, 57 males and 23 females in group B (mean age of 54.18 \pm 12.97 ), and 51 males and 29 females in group C (mean age of 55.59 \pm 11.88 ). The courses of diseases were $11.40 \pm 6.64,10.70 \pm 6.96$ and $12.20 \pm 6.01$, respectively, in group A, B and C. There appeared to be only minor differences regarding the age, sex ratio and diseases duration before treatment among the three groups (all $\mathrm{P}>0.05$ ). Furthermore, no case was eliminated during the treatment period.

Comparison of indices. FPG, HbA1c and $2 \mathrm{~h}$ blood glucose all indicated significant decreased tendency among the three groups after treatment than those before treatment, and was the most significant in group $\mathrm{C}$ (all $\mathrm{P}<0.05)$. Little evident statistical distinction was found between groups $\mathrm{A}$ and $\mathrm{B}$ with respect to the glucose indices $(\mathrm{P}>0.05)$. Besides, triglyceride, cholesterol, HDL and LDL were also suppressed later in the three groups when contrasted with the indices before treatment, significantly decreased tend was found in group C. Furthermore, alanine aminotransferase, aspartate aminotransferase, serum total bilirubin, direct bilirubin were decreased after treatment among groups $\mathrm{A}-\mathrm{C}$ (all $\mathrm{P}<0.05)$, but without statistical distinction between groups $\mathrm{A}$ and $\mathrm{B}(\mathrm{P}>0.05)$ (Table I).

Compared with values before treatment, the BMI, waist circumference and waist/hip ratio decreased significantly in group $\mathrm{C}$ than those of the before treatment levels (all $\mathrm{P}<0.05$ ), but showed no apparent changes in the other two groups ( $\mathrm{P}>0.05)$. Besides, the BMI, waist circumference and waist/hip ratio decreased obviously in group $\mathrm{C}$ after treatment than in groups $\mathrm{A}$ and $\mathrm{B}$, with statistical significance (both $\mathrm{P}<0.05$ ), 
but there was no statistical difference regarding the BMI, waist circumference and waist/hip ratio after treatment between groups $\mathrm{A}$ and $\mathrm{B}(\mathrm{P}>0.05)$.

Comparison of serum adiponectin level. Compared with before treatment, the serum amount of adiponectin increased tremendously in the three groups after treatment (all $\mathrm{P}<0.05$ ), while levels in the group $\mathrm{C}$ was significantly higher than the levels of groups A and B (both $\mathrm{P}<0.05$ ). However, little evident statistical distinction was found between groups A and B regarding serum adiponectin levels after treatment (Table I).

Included studies. Finally, a total of 6 cohort studies were reasonable for the present meta-analysis (4 Asian-based and 2 Caucasian-based studies) metformin between 2006 and 2014 , including 267 subjects with T2DM altogether (25-28,30,31). All quality results of the included studies were in moderate to high grade, as shown in Fig. 1.

Association of combination therapy with serum adiponectin level. As shown in Fig. 2A, after treatment serum adiponectin level in T2DM sufferers appeared greater than pretreatment level in those sufferers (SMD $=1.27,95 \%$ CI: 0.73-1.81, $\mathrm{P}<0.001)$. Subgroup analysis acccording to ethnicity indicated that combination therapy of metformin and rosiglitazone may increase the level of serum adiponectin in T2DM patients among Asians and Caucasians (both $\mathrm{P}<0.05$ ) (Fig. 2B-D). By using both RIA and ELISA, an increased serum adiponectin level was detected after the treatment of metformin and rosiglitazone in sufferers of T2DM (both $\mathrm{P}<0.001$ ). Further, the serum level of adiponectin in T2DM sufferers after treatment was greater than the level in the sufferers before treatment in both $\leq 6$ months and $>6$ months subgroups (both $\mathrm{P}<0.001$ ).

Sensitivity analysis as well as publication bias. Sensitivity analysis suggested that there was no single study that could have an adverse effect on the overall estimation of the present analyses (Fig. 3A). The graphical funnel plots of those 6 cases appeared symmetrical, and Egger's test implied no publication bias ( $\mathrm{t}=0.96, \mathrm{P}=0.368)$ (Fig. 3B).

Further analysis. This study found that the combination of metformin as well as rosiglitazone has roles in increasing serum level of adiponectin involving in improving glucose and lipid metabolism, liver function indices, as well as body fat mass control, suggesting that metformin plus rosiglitazone therapy is a suitable choice for treating T2DM clinically. The results were confirmed both in the clinic-based cohort study and meta-analysis of this study.

Many types of well-established anti-diabetic drugs exist including metformin, glimepiride and rosiglitazone, also termed as triple-therapy for T2DM; the experimental results manifested that the triple-therapy can significantly increase adiponectin serum levels compared to the combination of metformin + glimepiride, and is efficacious in lessening diabetic cardiomyopathy (32). As presented in this study, the effects of single metformin or rosiglitazone treatment showed significantly lowered efficacies in decreasing glucose and lipid metabolism, liver function, as well as body fat mass indices, which eventually confirmed the combination effect

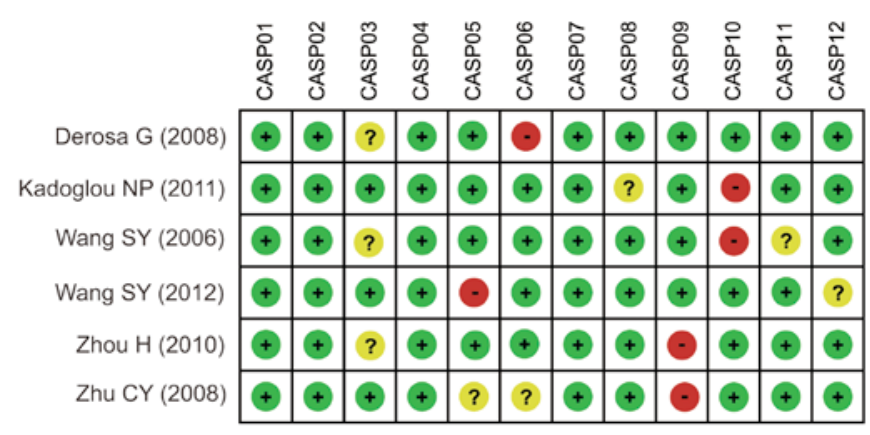

Figure 1. Methodological quality assessment according to the CASP Green, yes; red, no; yellow, unknown. CASP, Critical Appraisal Skills Programme.

of metformin or rosiglitazone regimens in treating T2DM. The combined therapy of metformin plus rosiglitazone obtained improvement of lipid and glucose metabolism and insulin resistance reduction, as it was previously reported, which elucidating the positive functions of this therapy through downregulating leptin levels and elevating serum levels of adiponectin (33). Importantly and concretely, with respect to the interaction mechanism between metformin and rosiglitazone that have led to the significant improvement in serum adiponectin level, it was considered that adiponectin elevated insulin sensitivity via the stimulation of glucose utilization and increasing free fatty acid oxidation based on the adenosine monophosphate-activated protein kinase (AMPK) signal pathway, besides, PPAR- $\gamma$ response element (PPRE) is present in the promoter region of adiponectin gene, suggesting an important role of PPAR- $\gamma$ in regulating the synthesis of adiponectin gene $(34,35)$. Based on the above description of the effect of metformin in modulating AMPK and involving the IR regulation, as well as the pharmacological property of rosiglitazone as one of the compounds of thiazolidinedione, acting as a selective agonist of PPAR- $\gamma(36,37)$, such combination may significantly promote the synthesis and secretion of adiponectin contributing to increasing level of adiponectin and improving insulin sensitivity. Another published study demonstrated that T2DM patients suffer the complication of cardiovascular disease, however, the oral intake of metformin plus rosiglitazone was useful for alleviating hyperglycemic syndrome and reducing blood pressure by mediating glucose uptake in skeletal muscle and increasing adiponectin expression to assist insulin activities (38).

In view of previous opinions from multiple aspects, we put forward that the relationship between metformin plus rosiglitazone application and adiponectin levels in T2DM patients is significant and this drug therapy is advantageous to suppress hepatic glycogenesis and increase adiponectin level to enhance insulin sensitivity, which effectively relieve weight gain and blood pressure. Li et al came up with an opinion in line with our study result that the combining therapy of metformin and rosiglitazone is a more effective method for treating T2DM compared with metformin monotherapy and the dominant effects resulted in insulin release, different normal $\beta$-cell function, thereby improving body fat and ameliorating T2DM severity (39). In addition, further meta-analysis also confirmed the results that combination of metformin and rosiglitazone treatment increased serum adiponectin levels in a majority of 
A

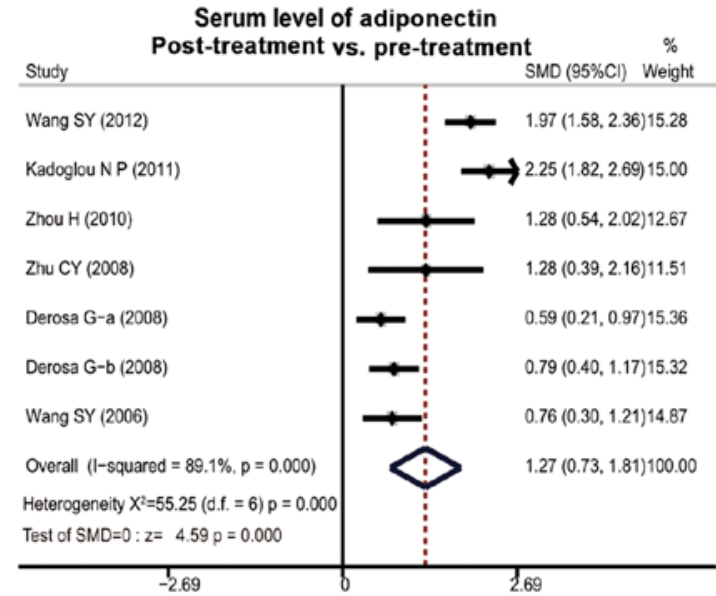

$\mathrm{C}$

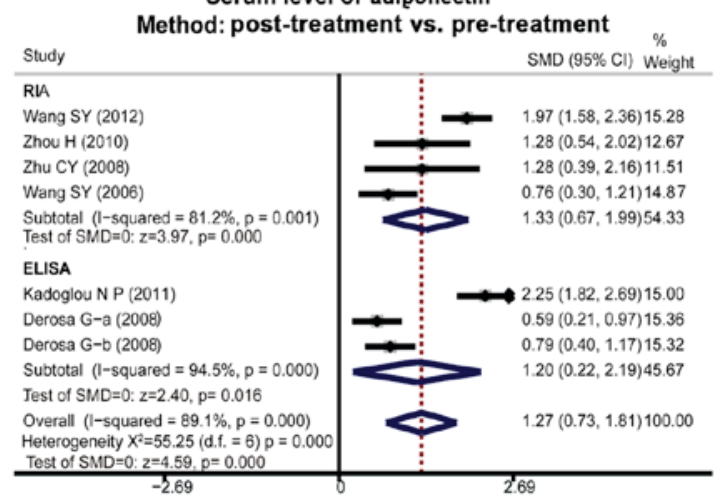

Serum level of adiponectin Ethnicity: post-treatment vs. pre-treatment

\begin{tabular}{|c|c|}
\hline Study & SMD $(95 \%$ Cl) Weight \\
\hline Asians & \\
\hline Wang SY (2012) & $1.97(1.58 .2 .36) 15.28$ \\
\hline Zhou H (2010) & $1.28(0.54,2.02) 12.67$ \\
\hline Zhu $\mathrm{CY}(2008)$ & $1.28(0.39,2.16) 11.51$ \\
\hline Wang SY (2006) & $0.76(0.30,1.21) 14.87$ \\
\hline $\begin{array}{l}\text { Subtotal (I-squared }=81.2 \%, p=0.001 \text { ) } \\
\text { Test of } \operatorname{SMD}=0: z=3.97, p=0.000\end{array}$ & $1.33(0.67,1.99) 54.33$ \\
\hline Caucasians & \\
\hline Kadoglou N P (2011) & $2.25(1.82 .2 .69) 15.00$ \\
\hline Derosa G-a (2008) & $0.59(0.21,0.97) 15.36$ \\
\hline Derosa G-b (2008) & $0.79(0.40,1.17) 15.32$ \\
\hline $\begin{array}{l}\text { Subtotal }(1-\text { squared }=94.5 \%, p=0.000) \\
\text { Test of } S M D=0: z=2.40, p=0.016\end{array}$ & $1.20(0.22,2.19) 45.67$ \\
\hline $\begin{array}{l}\text { Overall (I-squared }=89.1 \%, p=0.000) \\
\text { Heterogeneily } X^{2}=55.25(d f .=6) p=0.000 \\
\text { Test of } S M D=0: z=4.59, p=0.000\end{array}$ & $1.27(0.73,1.81) 100.00$ \\
\hline
\end{tabular}

D Serum level of adiponectin Follow up: post-treatment vs. pre-treatment

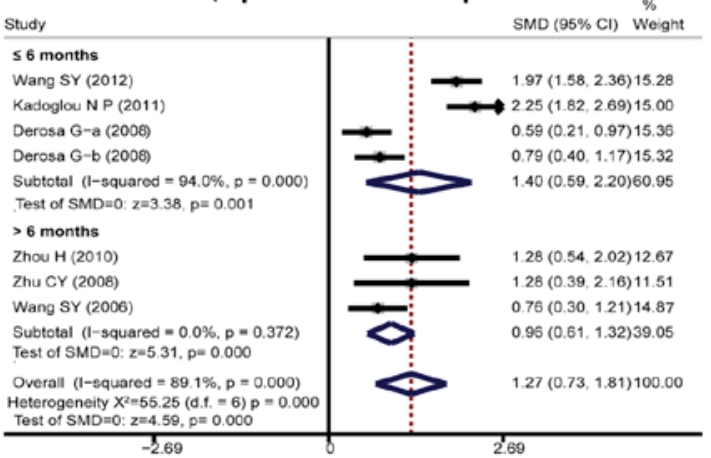

Figure 2. (A) Forest plots for the relationships exploration of the effects of metformin plus rosiglitazone on serum adiponectin levels in sufferers of T2DM. (B-D) Subgroup discussion by ethnicity, detection methods and continuation exploring the effects of metformin plus rosiglitazone on serum adiponectin levels in sufferers of T2DM. T2DM, type 2 diabetes mellitus.

A

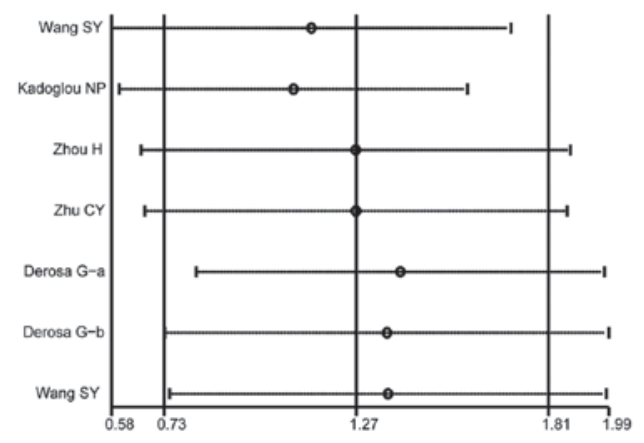

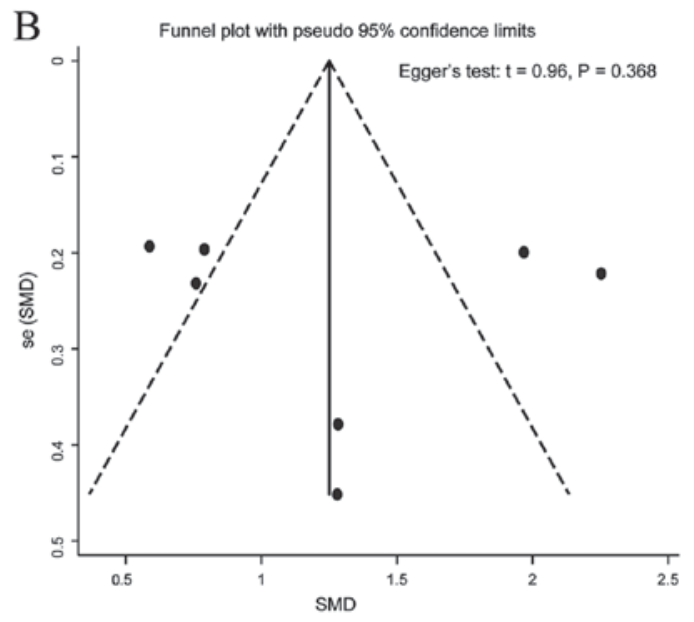

Figure 3. (A) Sensitivity analysis performance assessing whether single study could affect the whole results in our meta-analysis. (B) Publication bias exploration of the summary standard mean differences coefficients based on investigating the effects of metformin plus rosiglitazone on serum adiponectin levels in sufferers of T2DM. T2DM, type 2 diabetes mellitus.

subjects, suggesting that metformin plus rosiglitazone therapy is a suitable choice for the treatment of T2DM.

In conclusion, our findings revealed that combination of metformin and rosiglitazone increased serum level of adiponectin, suggesting that metformin plus rosiglitazone therapy is a suitable choice for the treatment of T2DM. Importantly, continuous studies with large sample size evaluating the biological significance of concrete adiponectin 
levels as well as other sensitive biomarkers in T2DM patients receiving metformin and rosiglitazone or other medicines are recommended in future studies.

\section{References}

1. Garrett CR, Hassabo HM, Bhadkamkar NA, Wen S, Baladandayuthapani V, Kee BK, Eng C and Hassan MM: Survival advantage observed with the use of metformin in patients with type II diabetes and colorectal cancer. Br J Cancer 106: 1374-1378, 2012.

2. Zhai J, Liu CX, Tian ZR, Jiang QH and Sun YP: Effects of metformin on the expression of GLUT4 in endometrium of obese women with polycystic ovary syndrome. Biol Reprod 87: 29, 2012.

3. Hindlet P, Barraud C, Boschat L, Farinotti R, Bado A and Buyse M: Rosiglitazone and metformin have opposite effects on intestinal absorption of oligopeptides via the proton-dependent PepT1 transporter. Mol Pharmacol 81: 319-327, 2012.

4. Cabreiro F, Au C, Leung KY, Vergara-Irigaray N, Cochemé HM, Noori T, Weinkove D, Schuster E, Greene ND and Gems D: Metformin retards aging in $C$. elegans by altering microbial folate and methionine metabolism. Cell 153: 228-239, 2013.

5. Cappelli C, Rotondi M, Pirola I, Agosti B, Formenti A Zarra E, Valentini U, Leporati P, Chiovato L and Castellano M Thyreotropin levels in diabetic patients on metformin treatment. Eur J Endocrinol 167: 261-265, 2012.

6. Cheung BM: Behind the rosiglitazone controversy. Expert Rev Clin Pharmacol 3: 723-725, 2010.

7. He H, Tao H, Xiong H, Duan SZ, McGowan FX Jr, Mortensen RM and Balschi JA: Rosiglitazone causes cardiotoxicity via peroxisome proliferator-activated receptor $\gamma$-independent mitochondrial oxidative stress in mouse hearts. Toxicol Sci 138: 468-481, 2014.

8. Zhou M, Xu A, Lam KS, Tam PK, Che CM, Chan L, Lee IK, Wu D and Wang Y: Rosiglitazone promotes fatty acyl CoA accumulation and excessive glycogen storage in livers of mice without adiponectin. J Hepatol 53: 1108-1116, 2010

9. Bach RG, Brooks MM, Lombardero M, Genuth S, Donner TW, Garber A, Kennedy L, Monrad ES, Pop-Busui R, Kelsey SF, et al; BARI 2D Investigators: Response to letter regarding article, 'rosiglitazone and outcomes for patients with diabetes mellitus and coronary artery disease in the Bypass Angioplasty Revascularization Investigation 2 Diabetes (BARI 2D) Trial'. Circulation 129: e460-e461, 2014.

10. Gokulakrishnan K, Aravindhan V, Amutha A, Abhijit S, Ranjani H, Anjana RM, Unnikrishnan R, Miranda P, Narayan KM and Mohan V: Serum adiponectin helps to differentiate type 1 and type 2 diabetes among young Asian Indians. Diabetes Technol Ther 15: 696-702, 2013.

11. Kuo JZ, Guo X, Klein R, Klein BE, Genter P, Roll K, Hai Y, Goodarzi MO, Rotter JI, Chen YD, et al: Adiponectin, insulin sensitivity and diabetic retinopathy in Latinos with type 2 diabetes. J Clin Endocrinol Metab 100: 3348-3355, 2015.

12. Sun WL, Chen LL, Zhang SZ, Wu YM, Ren YZ and Qin GM: Inflammatory cytokines, adiponectin, insulin resistance and metabolic control after periodontal intervention in patients with type 2 diabetes and chronic periodontitis. Intern Med 50: 1569-1574, 2011.

13. Dalmas E, Venteclef N, Caer C, Poitou C, Cremer I, Aron-Wisnewsky J, Lacroix-Desmazes S, Bayry J, Kaveri SV, Clément K, et al: T cell-derived IL-22 amplifies IL-1 $\beta$-driven inflammation in human adipose tissue: Relevance to obesity and type 2 diabetes. Diabetes 63: 1966-1977, 2014.

14. Akash MS, Rehman K and Chen S: Role of inflammatory mechanisms in pathogenesis of type 2 diabetes mellitus. J Cell Biochem 114: 525-531, 2013.

15. Donath MY and Shoelson SE: Type 2 diabetes as an inflammatory disease. Nat Rev Immunol 11: 98-107, 2011.

16. Ando T, Ishikawa $\mathrm{T}$, Takagi $\mathrm{T}$, Imamoto $\mathrm{E}$, Kishimoto $\mathrm{E}$, Okajima A, Uchiyama K, Handa O, Yagi N, Kokura S, et al: Impact of Helicobacter pylori eradication on circulating adiponectin in humans. Helicobacter 18: 158-164, 2013.

17. Jeong HG, Min BJ, Lim S, Kim TH, Lee JJ, Park JH, Lee SB, Han JW, Choi SH, Park YJ, et al: Plasma adiponectin elevation in elderly individuals with subsyndromal depression. Psychoneuroendocrinology 37: 948-955, 2012.

18. Abdelgadir M, Karlsson AF, Berglund L and Berne C: Low serum adiponectin concentrations are associated with insulin sensitivity independent of obesity in Sudanese subjects with type 2 diabetes mellitus. Diabetol Metab Syndr 5: 15, 2013.
19. Esteghamati A, Mansournia N, Nakhjavani M, Mansournia MA, Nikzamir A and Abbasi M: Association of $+45(\mathrm{~T} / \mathrm{G})$ and $+276(\mathrm{G} / \mathrm{T})$ polymorphisms in the adiponectin gene with coronary artery disease in a population of Iranian patients with type 2 diabetes. Mol Biol Rep 39: 3791-3797, 2012.

20. Ouchi N, Parker JL, Lugus JJ and Walsh K: Adipokines in inflammation and metabolic disease. Nat Rev Immunol 11: 85-97, 2011.

21. Zulian A, Cancello R, Girola A, Gilardini L, Alberti L, Croci M, Micheletto G, Danelli P and Invitti C: In vitro and in vivo effects of metformin on human adipose tissue adiponectin. Obes Facts 4: 27-33, 2011

22. Singh S, Akhtar N and Ahmad J: Plasma adiponectin levels in women with polycystic ovary syndrome: Impact of metformin treatment in a case-control study. Diabetes Metab Syndr 6: 207-211, 2012.

23. Tao L, Wang Y, Gao E, Zhang H, Yuan Y, Lau WB, Chan L, Koch WJ and Ma XL: Adiponectin: An indispensable molecule in rosiglitazone cardioprotection following myocardial infarction. Circ Res 106: 409-417, 2010.

24. Mahgoub H, Youssef RM, Korany MA, Khamis EF and Kamal MF: Development and validation of spectrophotometric and HPTLC methods for simultaneous determination of rosiglitazone maleate and metformin hydrochloride in the presence of interfering matrix excipients. Drug Dev Ind Pharm 40: 1190-1198, 2014.

25. Wang SY and Shen TT: Observation of curative effect of rosiglitazone combined with metforminon type 2 diabetes mellitus. Mod J Integr Tradit Chin West Med 21: 1046-1047, 2012 (In Chinese).

26. Wang SF, Qu XH and Zhao Y: Clinical observation of therapeutic efficacy of insulin sensitizer in treatment of newly-diagnosed type 2 diabetes. Chin J Diabetes 14: 415-416, 2006 (In Chinese).

27. Zhou H, Teng XY and Liu W: Clinical analysis of combined therapy of rosiglitazone and metforminon in trahepatic fat deposition in type 2 diabetic patients. J Shanghai Jiaotong Univ 30: 1263-12666, 2010 (In Chinese).

28. Zhu CY, Zhou H, Han Y, Ling LQ and Huang G: Application of adiponectin, tumor necrosis factor alpha and ferritin determination in type 2 diabetes mellitus with fatty liver. Nucl Technol 31: 356-359, 2008.

29. American Diabetes Association: Standards of medical care in diabetes - 2007. Diabetes Care 30 (Suppl 1): S4-S41, 2007.

30. Kadoglou NP, Kapelouzou A, Tsanikidis H, Vitta I, Liapis CD and Sailer N: Effects of rosiglitazone/metformin fixed-dose combination therapy and metformin monotherapy on serum vaspin, adiponectin and IL-6 levels in drug-naïve patients with type 2 diabetes. Exp Clin Endocrinol Diabetes 119: 63-68, 2011.

31. Derosa G, Salvadeo SA, D'Angelo A, Fogari E, Ragonesi PD, Ciccarelli L, Piccinni MN, Ferrari I, Gravina A, Maffioli P, et al: Rosiglitazone therapy improves insulin resistance parameters in overweight and obese diabetic patients intolerant to metformin. Arch Med Res 39: 412-419, 2008.

32. Sharma AK and Srinivasan BP: Triple verses glimepiride plus metformin therapy on cardiovascular risk biomarkers and diabetic cardiomyopathy in insulin resistance type 2 diabetes mellitus rats. Eur J Pharm Sci 38: 433-444, 2009.

33. Derosa G, Fogari E, Cicero AF, D'Angelo A, Ciccarelli L, Piccinni MN, Pricolo F, Salvadeo SA, Gravina A, Ferrari I, et al: Blood pressure control and inflammatory markers in type 2 diabetic patients treated with pioglitazone or rosiglitazone and metformin. Hypertens Res 30: 387-394, 2007.

34. Chen H, Zhang L, Li X, Li X, Sun G, Yuan X, Lei L, Liu J, Yin L, Deng Q, et al: Adiponectin activates the AMPK signaling pathway to regulate lipid metabolism in bovine hepatocytes. J Steroid Biochem Mol Biol 138: 445-454, 2013.

35. Ahmadian M, Suh JM, Hah N, Liddle C, Atkins AR, Downes M and Evans RM: PPAR $\gamma$ signaling and metabolism: The good, the bad and the future. Nat Med 19: 557-566, 2013.

36. Kristensen JM, Treebak JT, Schjerling P, Goodyear L and Wojtaszewski JF: Two weeks of metformin treatment induces AMPK-dependent enhancement of insulin-stimulated glucose uptake in mouse soleus muscle. Am J Physiol Endocrinol Metab 306: E1099-E1109, 2014.

37. Yang DR, Lin SJ, Ding XF, Miyamoto H, Messing E, Li LQ, Wang $\mathrm{N}$ and Chang $\mathrm{C}$ : Higher expression of peroxisome proliferator-activated receptor gamma or its activation by agonist thiazolidinedione-rosiglitazone promotes bladder cancer cell migration and invasion. Urology 81: 1109.e1-e6, 2013.

38. Bailey CJ: Treating insulin resistance in type 2 diabetes with metformin and thiazolidinediones. Diabetes Obes Metab 7: 675-691, 2005.

39. Li X, Zhang N,Li Y, Shi Y,Li D, Xie Y, Xie Y and Ming J: Effects of metformin and rosiglitazone on peripheral insulin resistance and $\beta$-cell function in obesity: A double-blind, randomized, controlled study. J Int Med Res 39: 358-365, 2011. 